\title{
Enfermedad, eutanasia y aborto: una reflexión desde la bioética
}

Yelson Alejandro Picón Jaimes a ; Javier Esteban Orozco Chinome b; Iván David Lozada Martínez'; Luis Rafael Moscote Salazar ${ }^{\text {d }}$

a Médico. Especialista en bioética aplicada al campo clínico y experimental. Magíster en bioética. ORCID: https://orcid.org/0000-0002-7498-5346, ypicon@ unab.edu.co ; b Médico cirujano. Magíster en medicina regenerativa y antienvejecimiento. ORCID: https://orcid.org/0000-0001-6013-3997, efeso17@ yahoo. es; ${ }^{\mathbf{c}}$ Estudiante de Medicina. Investigador. Universidad de Cartagena. ORCID: https://orcid.org/0000-0002-1960-7334, ivandavidloma@ gmail.com ; ${ }^{\mathrm{d}}$ Médico. Neurocirujano. Investigador de la Universidad de Cartagena. ORCID: https://orcid.org/0000-0002-4180-6962, rafaelmoscote21@ gmail.com DOI: https://doi.org/10.22517/25395203.24659

\section{Resumen}

El objetivo de este manuscrito es reflexionar sobre la enfermedad, la eutanasia y el aborto desde la bioética, considerando las implicaciones legales en Colombia y la visión al respecto de profesionales dedicados a las ciencias de la salud en diferentes lugares del mundo. Las enfermedades crónicas son causa importante de limitación funcional, sufrimiento y mortalidad; algunas patologías comprometen tanto el estado de conciencia en las personas que afectan la capacidad de decidir, por lo cual, es importante buscar alternativas para garantizar la autonomía de los pacientes. La eutanasia es aceptada solo en algunos países, pero cuenta con dificultades para el acceso, debido a trámites burocráticos o falta de reglamentación. Finalmente, el aborto es un procedimiento altamente cuestionado por colectivos sociales y religiosos, lo cual ha llevado a la estigmatización de las mujeres que desean acceder a él.

Palabras clave: Bioética, Enfermedad crónica, Eutanasia, Aborto

\section{Disease, euthanasia and abortion: a reflection from bioethics}

\begin{abstract}
This manuscript aims to reflect on the disease, euthanasia, and abortion from bioethics, reviewing the legal implications in Colombia and comparing with the vision of health sciences professionals in different parts of the world. Chronic diseases are a major cause of functional limitation, suffering, and mortality. Some pathologies compromise the state of consciousness in people, which affects the ability to decide, so it is important to look for alternatives to guarantee the autonomy of patients. Euthanasia is accepted in only a few countries, but it has difficulties with access due to red tape or lack of regulation. Finally, abortion is judged by religious and social groups, which has led to the stigmatization of women who wish to access it.
\end{abstract}

Key words: Bioethics, Chronic disease, Euthanasia, Abortion

\section{Introducción}

La toma de decisiones es un proceso de pensamiento complejo donde se busca la elección de una forma de actuar frente a una determinada situación a través de dos métodos, el primero de ellos es el pensamiento intuitivo que es rápido, automatizado, compuesto por emociones y sin esfuerzo; el segundo, es el pensamiento deliberado, que es lento, controlado, detallista, minucioso y neutro en cuanto a emociones (1),(2). En la práctica clínica, la toma de decisiones se ha vuelto una actividad de gran importancia, por lo cual, áreas como la bioética surgieron para acompañar el proceso de elección ante situaciones que ponen en riesgo la integridad de los seres humanos, en momentos donde existe la necesidad de escoger un camino que respete la vida como bien superior frente a las posibilidades dadas por el desarrollo de la ciencia médica (3).

En la actualidad, son muchas las situaciones que requieren de un esfuerzo decisivo, pues los humanos son tan diversos como los pensamientos que circulan sus mentes, siempre con la posibilidad de tener ideas diferentes frente a los escenarios que se plantean en el diario vivir.

Este manuscrito está dirigido a los profesionales de la salud, que durante el desarrollo de sus labores ven la necesidad de acompañar a los pacientes y participar de las decisiones de estos, enmarcados en el ámbito de la relación profesional médico-paciente. 
Inicialmente, se abordará el tema de la enfermedad crónica ya que según datos de la organización mundial de salud OMS, cada año en el mundo fallecen 30 millones de personas por patologías crónicas no transmisibles, y en Colombia, la cifra alcanza 110 mil fallecimientos por enfermedades como el cáncer, los trastornos cardiovasculares y la patología pulmonar (4). En cuanto a la legislación colombiana, se destacan la ley 1355 de 2009 por medio de la cual se fija a la obesidad como una enfermedad crónica de importancia para la salud pública, siendo causa directa de trastornos como las patologías cardiovasculares, la depresión, la diabetes y de alteraciones osteomusculares que aumentan las tasas de mortalidad a nivel nacional (5). Otra ley importante es la 1733 de 2014, por medio de la cual se regulan los servicios de cuidados paliativos para el manejo integral de pacientes con enfermedades terminales, crónicas, degenerativas e irreversibles en cualquier fase, con el objetivo de mitigar el alto impacto en la calidad de vida (6).

Posteriormente, se tratará la cuestión bioética sobre la eutanasia; teniendo en cuenta que en el mundo solo en 5 países la han regulado, entre ellos Colombia, y cuatro más han establecido el suicidio asistido para personas con patologías incurables. El tema a nivel nacional ha sido apoyado desde 1997 por la corte constitucional, reglamentándose en 2015 e instando a las entidades promotoras de salud a garantizar el acceso a este tratamiento, e incluso se ha permitido para menores de edad; sin embargo, el proyecto de ley no ha superado los debates en el congreso de la República, por tanto no se cuenta con una ley que regule dicha práctica (7).

Finalmente, se tratará el aborto. Actualmente, en Colombia, este proceso está regulado bajo la premisa de interrupción voluntaria del embarazo, mediante la sentencia de la corte constitucional C-355 de 2006 en tres causales: 1) cuando la gestación implica peligro para la vida o la salud de la mujer, 2) cuando existen malformaciones fetales graves, incompatibles con la vida y 3) cuando la gestación no es consentida por la mujer, como en el caso del acceso carnal violento o el uso de técnicas de reproducción asistida no informadas (8).

El objetivo de este manuscrito consiste en reflexionar, de manera crítica, sobre la enfermedad crónica, la eutanasia y el aborto desde la bioética, revisando las implicaciones legales en Colombia y haciendo una comparación con la visión de profesionales dedicados a las ciencias de la salud en diferentes lugares del mundo.

\section{La enfermedad crónica}

La enfermedad se define por oposición al concepto de salud. Para algunos sectores gubernamentales, la salud es el equilibrio de condiciones óptimas e ideales, excluyentes de la situación de enfermedad, definición que plantea una dicotomía entre tener bienestar o no tenerlo. Por otro lado, otros autores consideran que el proceso salud-enfermedad es dinámico, cambiante y no necesariamente, excluyente (9).

Tenjo-Cogollo (10), por ejemplo, considera la visión teológica de la salud y explica como en textos antiguos como el Evangelio de Lucas "Pasión y muerte de Jesús", se evidencia una clara lucha de las personas contra el sufrimiento y la búsqueda de una explicación para este (10). Para el autor, la enfermedad es una entidad muy temida universalmente por los humanos y suele lucharse contra ella, llegando incluso a posiciones radicales como los tratamientos extremos para preservar la vida, o, todo lo contrario, personas que asumen la idea de muerte desde el inicio del diagnóstico de la enfermedad (10).

La literatura médica revela importantes investigaciones llevadas a cabo por científicos en todos los rincones del mundo sobre las distintas patologías y se establecen las que se consideran como enfermedades crónicas con gran afectación para los individuos como la Esclerosis Lateral Amiotrófica (ELA), por citar un ejemplo. La ELA es una entidad neurodegenerativa, rápidamente progresiva que puede terminar en una limitación funcional total para quien la padece, causando que la toma de decisiones para el profesional de la salud y para el paciente se empieza a ver conflictuada: $¿$ Es suficiente poner al individuo en la categoría de "incurable" y dejar que la naturaleza siga su curso?, o ¿Se deben realizar esfuerzos para mantener la vida, recordando el caso del el gran científico Stephen Hawking, quien padeció esta enfermedad y vivió hasta los 76 años? Al respecto, Scull-Torres (11) en 2019 publicó un trabajo cualitativo titulado "La observancia de la bioética en el cuidado paliativo de enfermos con ELA", donde concluyó que la universalidad de los principios bioéticos que hoy son ampliamente difundidos, es limitada por la legislación de cada país y depende prácticamente de lo que puede ser considerado apto o no en cada región, por tanto, se cuestionó la universalidad de los principios de la bioética. Adicionalmente, mencionó la importancia de educar, más que de informar al paciente sobre el proceso de la enfermedad, lo cual consigue decisiones moralmente mejor aceptadas entre el individuo, su familia y su tratante, permitiendo hasta donde sea posible el ejercicio de la autonomía responsable y cuando esta ya no puede ser ejercida por el sujeto, se debe procurar al máximo 
que las decisiones por sustitución reflejen los valores de la persona enferma (11).

Para Toro-flores et al (12) el diligenciamiento de un formato de decisiones anticipadas, en definitiva, facilita la toma de decisiones en salud, especialmente en casos en que las patologías terminan por afectar las funciones cognitivas superiores de los sujetos, si bien es un proceso poco conocido por los pacientes.

En síntesis, las enfermedades crónicas pueden comprometer el bienestar del individuo a niveles que incluso en muchas situaciones vulneran la dignidad misma de la persona, por eso, es importante buscar instrumentos que garanticen la mitigación del daño a la esencia del individuo. Estas estrategias dependen de la legislación vigente para cada país.

Para el caso colombiano, una buena maniobra es la elaboración del documento de voluntades anticipadas que se rige legalmente a través de la Resolución 2665 de 2018, en la cual se establecen los parámetros para la elaboración del citado documento, que puede ser, por escrito, audio o video, frente a notario, dos testigos o expresada al médico tratante. Si bien es un documento accesible, planteado desde muchas perspectivas para que sea práctico, e incluso pueda ser revocado; hay un gran desconocimiento entre los usuarios de los servicios de salud sobre su existencia. La invitación es querespetando un pilar fundamental para la bioética como lo es la dignidad, el respeto a la voluntad del individuo y la autonomía- se deben fortalecer en los profesionales de la salud las competencias para exponer a los pacientes la existencia de los documentos de voluntades anticipadas, para así, facilitar la toma de decisiones, velando siempre por el respeto a la dignidad de la persona.

\section{Eutanasia}

El final de la vida es otro de esos procesos que generan temor e inconformidad, especialmente entre los adultos mayores, pues las enfermedades crónicas son una importante causa de disrupción del proceso saludenfermedad que se acompaña de dolor y padecimiento (13). Es importante abordar el controversial término "Eutanasia", que es definido por Malespina (14), como el acto de matar a otra persona por petición suya para aliviarle el sufrimiento; pero, su texto no se limita a esa cruda definición, ya que menciona que en el derecho positivo, los estados que regulan este procedimiento como Bélgica, Holanda y Luxemburgo, exigen que sea llevado a cabo por un galeno, que debe estar convencido de que el padecimiento del individuo es insoportable y sin opciones de mejoría alguna (14).
En este punto, entran en juego valores como la libertad, la autonomía y la dignidad que son ampliamente defendidos por el derecho positivo en el ámbito internacional, pero que en su definición pueden caer en un plano netamente utilitarista, permitiendo ejercer estos valores pensando solamente en la consecuencia y dejando de lado la propia esencia del ser (14). Una vez más surge la encrucijada en torno a la decisión, que para no dar más vueltas al asunto debe ser la mejor aceptada moralmente, según el contexto, según el paciente, sus familiares, sus creencias, pero limitados por la noción colectiva del estado y los modelos políticos regentes en cada región.

En Latinoamérica, en cuanto a la legislación sobre la eutanasia, países como Argentina cada vez tienen más procesos de judicialización relacionados con la intervención médica de la muerte. Alonso (15), en 2016 publicó un manuscrito que revisó los fallos judiciales en este país, producidos entre 1975 y el 2015, encontrando múltiples casos en los que el personal sanitario ha acudido a tribunales para solicitar la realización de la eutanasia (15). Los hallazgos de Alonso indican que si bien la eutanasia no es considerada como primera opción por los pacientes, por cuestiones de credo y cultura, los usuarios que la han solicitado han sido apoyados por los juzgados que se han pronunciado a favor, permitiendo el desistimiento para la realización de intervenciones médicas, aun cuando hacerlo condujera a la muerte.

En Chile, según lo reportado en 2016 por Espinoza et al (16), entre los enfermeros de cuidados paliativos del sistema sanitario público, se ha venido propagando una serie de actitudes positivas hacia el acompañamiento y soporte al final de la vida de los enfermos, lo cual indica un proceso de asimilación de la muerte como algo natural, que cada vez requiere de mayor conocimiento, para que se lleve a cabo con la mayor dignidad posible. Al respecto, Castejón et al (17) considera fundamental la capacitación del personal sanitario en todo lo relacionado con el final de la vida, pues indica que es una obligación ética estar preparado para afrontar las diversas situaciones, acompañar a la familia y evitar el ensañamiento terapéutico.

En el caso colombiano, único país latinoamericano que despenalizó la eutanasia desde la década de los 90's, los pacientes aún siguen enfrentando dificultades al momento de optar por este procedimiento, ya que inicialmente deben ser evaluados por un comité ético asistencial, quien es el que determina si el paciente es elegible para tal efecto. Dentro de los lineamientos legales, en 2015 se decretó que son las entidades promotoras de salud, las que deben contar con un equipo multidisciplinario para tal fin, e incluso se ha aprobado que menores de 
edad puedan acceder a la eutanasia, siempre y cuando su condición medica cumpla con los criterios estipulados (18).

De forma adicional, el surgimiento de las actuales clínicas de dolor en el país, ha ofrecido la opción de sedación paliativa para aquellos enfermos que pesé a su condición médica no deciden optar por la eutanasia, pero que a través de medicamentos para el dolor, pueden tener un menor sufrimiento en el desenlace de sus patologías terminales; sin estar exceptos de críticas, rechazo y sanción por algunos grupos sociales y religiosos (18).

Recapitulando, las personas que pueden optar por este procedimiento en Colombia, son los enfermos mayores de edad o menores (en casos específicos) con enfermedades en fase terminal y con criterios clínicos de mal pronóstico; así como los enfermos en fase terminal de patologías oncológicas que estén en capacidad de decisión y que puedan expresar su autonomía de manera verbal o escrita.

La recomendación para los profesionales de la salud es que en sus entornos de trabajo reflexionen sobre la eutanasia, desde una posición profesional, sin temer generar conflicto pues es un deber ético mencionarlo a los pacientes e instruirles ya que la mejor forma de atender a un usuario parte de hacerle conocer sus derechos, aun cuando estos entren en conflicto con la visión personal del profesional; pues si realmente se quiere no hacer daño, hay que respetar las libertades ajenas y la capacidad de decisión de la persona.

\begin{abstract}
Aborto
El aborto es una de los temas más polémicos entre los credos, los partidos políticos e incluso entre movimientos sociales, pues hay autores que consideran que es una experiencia que deshumaniza a quien la vive, y hay quienes están a favor de la elección de la mujer sobre su propio cuerpo (19).
\end{abstract}

Muchos estados han legislado y creado políticas con directrices precisas para acceder al aborto, aun así, siguen existiendo contrapartes, como se nota en el trabajo de Valenzuela et al (20), donde la escuela de enfermería de la Universidad Católica de Chile, da su posición manifestando que pese a los proyectos de ley que despenalizaron parcialmente el aborto en ese país, en 2017 , los y las profesionales de enfermería de esa escuela, se abstienen de participar en dichos procedimientos, pues manifiestan que va en contra del fin último de su profesión (20).
Otro escenario complejo que involucra y entrelaza las situaciones descritas con anterioridad es el reportado por Menezes et al (21), que en su escrito menciona tres casos en los cuales la decisión se ha centrado en cuanto a mantener o no las funciones vitales de gestantes con muerte cerebral decretada, para permitir el desarrollo fetal (21). A lo largo de sus líneas deja ver que los casos fueron abordados de manera diferente, dependiendo en gran medida de la legislación de los países donde sucedieron los hechos; obteniendo como resultado en algunos lugares el cese de las medidas de soporte pese a la disputa entre médicos y familiares, reduciéndose al contexto legal de si el feto, es o no considerado sujeto de derecho.

En Colombia, como se mencionó previamente, es la sentencia C-355 de 2006 la que permite acceder a la interrupción voluntaria del embarazo en las tres causales enunciadas anteriormente. Los nuevos proyectos que han buscado despenalizar completamente el aborto, no han prosperado en el ejecutivo nacional. Sin embargo, es importante recordar que, de forma legal, son las entidades promotoras de salud y las instituciones prestadoras de servicios de salud, independientemente de que pertenezcan al régimen contributivo o subsidiado, las que están en la obligación de cumplir con la decisión de la corte constitucional colombiana; siendo responsables los profesionales de la salud, quienes deben redireccionar a sus usuarios, que cumplen los requisitos para tal fin y remitirlo al especialista pertinente dentro del mismo centro.

Cabe resaltar que la objeción de conciencia es una medida legal para que el profesional que lo considere, limite su actuar ante determinada situación, pero este mecanismo es individual y transversal, por tanto, un profesional objetor, lo será en todos los lugares donde ejerza su acción y esta limitado por el recurso de la urgencia, donde si no hubiese nadie más que realice el procedimiento, es responsabilidad del profesional disponible, aunque sea objetor de conciencia.

En resumen, es deber de los profesionales de la salud conocer los flujos de derivación ante el escenario de una posible interrupción voluntaria del embarazo, con la finalidad de disminuir el tiempo de espera para su valoración y eventual realización, disminuyendo la presentación de embarazos avanzados para interrupción, pues en caso de que se presenten, la legislación vigente avala la interrupción siempre y cuanto quepa dentro de las tres causales. 


\section{Conclusiones}

La enfermedad continúa siendo asumida como un proceso catastrófico que genera gran preocupación en la población y que lleva a la toma de medidas desesperadas en el intento de conservar la salud. Los documentos de voluntades anticipadas son poco conocidos por las personas y pueden convertirse en una importante herramienta en la toma de decisiones ante un proceso de enfermedad con gran limitación funcional, a su vez, facilitan las decisiones a los familiares y a los profesionales de la salud. La eutanasia pese a ser aceptada o despenalizada en Colombia, no es del todo accesible y es vista como un acto que atenta contra las creencias y costumbres de algunos grupos sociales, por tanto, es importante continuar los procesos que lleven a su reglamentación, para que pueda ser ofrecida con justicia y garantizando siempre el respeto a la autonomía y la dignidad de la persona. Finalmente, sobre el aborto, en Colombia, se permite la interrupción del embarazo en las tres causales dictaminadas por la corte constitucional. $\mathrm{Al}$ respecto, debe de acompañarse a la mujer en la toma de decisiones sobre su cuerpo, para ello, es indispensable que el personal médico conozca las regulaciones estatales ya que si bien es un procedimiento aprobado en varios países, implica un trámite burocrático desgastante.

\section{Referencias}

1. Manrique H, de Castro-correa A. Toma de decisiones : intuición y deliberación en la experiencia de los decisores. Innovar. 2019; 29(73):149-64.

2. Patiño M. La dimensión religiosa de la vida cotidiana desde el proyecto filosófico de John Dewey. Ideas y Valores. 2019; 68(170):171-86.

3. De Souza L, De Oliveira L, Silva E, Siqueira-Batista R. Tomada de decisão em (bio)ética clínica: abordagens contemporâneas. Rev Bioética. 2016; 24(2):304-14.

4. Organización mundial de la salud. Las enfermedades no transmisibles (ENT), nuestro reto [Internet] 2020 [Consultado 16 de Enero de 2021]. Disponible en: https://www.paho.org/col/ index.php?option $=$ com content $\&$ view $=$ article $\&$ id $=1756$ : lasenfermedades-no-transmisibles-ent-nuestro-reto \&Itemid $=487$

5. World Health Organization. Policy - Ley 1355 de 2009. Por medio de la cual se define la obesidad y las enfermedades crónicas no transmisibles asociadas a esta como una prioridad de salud pública y se adoptan medidas para su control, atención y prevención. [Internet]2009 [Consultado 16 de Enero de 2021]. Disponible en: https://extranet.who.int/nutrition/gina/ en/node/26173

6. Congreso de la Republica de Colombia. Ley Consuelo Devis Saavedra "Ley 1733" [Internet]. Diario Oficial No.
49.268. 2014 [Consultado 16 de Enero de 2021]. Disponible en:http://www.secretariasenado.gov.co/senado/basedoc/ ley $1733 \quad 2014 . \mathrm{html}$

7. Lampert M. Aplicación de la Eutanasia: Bélgica, Colombia, Holanda y Luxemburgo [Internet]. Asesoría Técnica Parlamentaria. Biblioteca Nacional del Congreso de Chile. 2019 [Consultado 16 de Enero de 2021]. Disponible en: https://www.bcn.cl/obtienearchivo?id=reposi torio/10221/27089/1/BCN Eutanasia Belgica Colombia Holanda Luxemburgo FINAL.pdf

8. Ministerio de Salud y Protección Social- República de Colombia. Interrupción voluntaria del embarazo, un derecho humano de las mujeres [Internet]. ABECÉ, línea: salud materna - derecho a la maternidad elegida. 2016 [Consultado 16 de Enero de 2021]. Disponible en: https://www.minsalud. gov.co/sites/rid/Lists/BibliotecaDigital/RIDE/VS/PP/abcmaternidad-elegida.pdf

9. Gómez-arias R. La vida y el sufrimiento más allá del cuerpo. Rev Fac Salud Pública. 2018; 36(1):103-29.

10. Tenjo-Cogollo M. Acompañamiento a enfermos de difícil cura a partir de Lc 23,39-43. Theol Xaver. 2018; 68(186):3943.

11. Scull-Torres M. La observancia de la bioética en el cuidado paliativo de enfermos con esclerosis lateral amiotrófica. Pers Bioet. 2019; 23(1):111-21.

12. Toro-Flores R, López-González R, López-Muñoz J. Conocimientos y actitudes de los pacientes críticos y sus familiares respecto a las directivas anticipadas y la toma de decisiones al final de la vida. Enfermería Intensiva. 2017; 28(1):21-30.

13. Batista J, Santana B, Machado J, Assis J. Ortotanásia nas unidades de terapia intensiva: percepção dos enfermeiros. Rev Bioética. 2017; 25(1):158-67.

14. Malespina ML. Cuestiones jurídicas al final de la vida. Pers y Bioética. 2017; 21(2):243-58.

15. Alonso JP. El derecho a una muerte digna en Argentina: la judicialización de la toma de decisiones médicas en el final de la vida. Physis Rev Saúde Coletiva. 2016; 26(2):569-89.

16. Espinoza-Venegas M, Luengo-Machuca L, SanhuezaAlvarado O. Actitudes en profesionales de enfermería chilenos hacia el cuidado al final de la vida. Análisis multivariado. Aquichan. 2016; 16(4):430-46.

17. Castejón O, Hernández H, Núñez R, Bush S. Dilemas éticos en la práctica clínica en las unidades de cuidados intensivos y paros cardiorrespiratorios. Rev Med Hondur. 2019; 87(1):33-7.

18. Calderón V, Machado-Duque M, Machado-Alba J. Sedation at the end of life. Ces Med. 2016; 30(1):46-54. 
19. Aznar-Sala F. Análisis del aborto desde un punto de vista conceptual. Pers y Bioética. 2019; 23(1):34-48.

20. Vargas I, Valenzuela M, Barrios S, Campos S, Uribe C, González X, et al. La escuela de enfermería UC ante la ley que despenaliza el aborto: Responde si al cuidado de los vulnerables. Horiz enfermería. 2017; 28(1):51-60.

21. Menezes R, Luna N. Gestação e morte cerebral materna: decisões em torno da vida fetal. Interface - Comun Saúde, Educ. 2017; 21(62):629-39. 